\title{
A Review of Methods for Synthesis of Al Nanoparticles
}

\author{
HAMID REZA GHORBANI* \\ Department of Chemical Engineering, Qaemshahr Branch, \\ Islamic Azad University, Qaemshahr, Iran. \\ ${ }^{*}$ Corresponding author E-mail: hamidghorbani6@gmail.com \\ http://dx.doi.org/10.13005/ojc/300456
}

(Received: October 01, 2014; Accepted: November 20, 2014)

\begin{abstract}
The synthesis of metallic nanoparticles is an active area of academic and, more significantly, applied research in nanotechnology. Several methods have been introduced for the synthesis of these materials. The techniques for synthesizing aluminum nanoparticles can be divided into solidphase, liquid-phase and gas-phase processes. The solid-phase techniques include mechanical ball milling and mechanochemical, the liquid-phase techniques include laser ablation, exploding wire, solution reduction, and decomposition process, whereas the gas-phase processes include gas evaporation, exploding wire, and laser ablation process. This study is an attempt to present an overview of Al nanoparticles preparation by various methods.
\end{abstract}

Key words: Synthesis, Al Nanoparticles, Solid, Liquid, Gas

\section{INTRODUCTION}

Nanotechnology plays an increasingly crucial role in many key technologies of the new millennium. The application of nanoscale materials and structures, usually ranging from 1 to $100 \mathrm{~nm}$, is an emerging area of nanoscience and nanotechnology ${ }^{1}$. Nanoparticles show unique properties compared to the bulk metals therefore a lot of research work has been reported for the synthesis and applications of metal nanoparticles².

Some of these metals (particularly aluminum) are previously widely used in energetic material formulations. Aluminium nanoparticles are of interest to a variety of fields including pyrotechnic, propellant, and explosive industries. Aluminium powder has been added to a range of these compositions to increase their performance through raising reaction energies, flame temperatures, and increasing blast rates. Nanoparticles of aluminium are more favourable because of their high enthalpy of combustion and rapid kinetics which increase these reaction properties even further ${ }^{3}$. It is known that nano-sized aluminum particles is a new energetic material with very high reactivity because of large specific surface area, and is expected to be applied to a next generation propellant in the field of aerospace applications. The reactivity of aluminum nanoparticle depends on the particle diameter. It is reported that $30-50 \mathrm{~nm}$ aluminum nanoparticles are most sensitive ${ }^{4}$. 
Physical and chemical properties of aluminum (Al) and especially its nanoparticles, are favorable enough to make them applicable in a variety of applications such as alloy powder metallurgy parts for automobiles and aircrafts, heat shielding coatings of aircrafts, corrosion, resistant, conductive and heat reflecting paints, conductive and decorative plastics, soldering and termite welding, pyrotechnics and military applications (rocket fuel, igniter, smokes, and tracers) $)^{5}$. Nanoscale Al particles are also studied as high-capacity hydrogen storage materials ${ }^{6}$.

There has been an extraordinary growth in nanoscience and technology in recent years, mainly due to both the development of new techniques to synthesize nanomaterials and the accessibility of tools for the classification and manipulation of nanoparticles ${ }^{7}$. Production of nanoparticles requires understanding of the fundamentals of nanoscale chemistry and physics, and know-how to commercialize them. Broadly speaking, there are two approaches to nanoparticle production: top-down and bottom-up. The former makes a material decrease its size from large to nanoscale, whereas the latter produces nanomaterials by starting from the atomic level ${ }^{\text {. }}$.

Generally, metal nanoparticles can be prepared and stabilized by chemical, physical and biological methods; the chemical approach, such as chemical reduction, electrochemical techniques, photochemical reduction and pyrolysis and physical methods, such as Arc-discharge and physical vapor condensation (pvc) is used ${ }^{9,10}$. Living organisms have huge potential for the production of nanoparticles/nanodevices of wide applications ${ }^{11}$.

Nevertheless high purity powders and nanopowders of active metals such as $\mathrm{Al}$ are not easily synthesized in as much as their rapid oxidation occurs easily ${ }^{5}$. The small sizes of aluminum nanoparticles make them particularly susceptible to excessive oxidation while being stored prior to use. Typically, the thickness of an oxide coating on an aluminum particle ranges from 1.7 to $6.0 \mathrm{~nm}$, irrespective of the size of the particle. If the passivating coatings also have an affinity for the binder material, then mixing problems can be resolved as well.
Studies have shown that the size, morphology, stability and properties (chemical and physical) of the metal nanoparticles are strongly influenced by the experimental conditions, the kinetics of interaction of metal ions with reducing agents, and adsorption processes of stabilizing agent with metal nanoparticles. Hence, the design of a synthesis method in which the size, morphology, stability and properties are controlled has become a major field of interest ${ }^{9}$. This paper is an attempt to present an overview of Al nanoparticles preparation by various methods.

\section{Al nanoparticles Synthesis}

Synthesis of nanomaterials by a simple, low cost and in high yield has been a great challenge since the very early development of nanoscience ${ }^{12}$. The techniques for synthesizing aluminum nanoparticles can be divided into solidphase, liquid-phase and gas-phase processes. The solid-phase techniques include mechanical ball milling and mechanochemical, the liquid-phase techniques include laser ablation, exploding wire, solution reduction, and decomposition process, whereas the gas-phase processes include gas evaporation, exploding wire, and laser ablation process.

\section{Solid-phase synthesis Mechanical ball milling}

Mechanical milling as a solid state synthesis usually performed using ball milling equipments that generally divided to "low energy" and "high energy" category based on the value of induced the mechanical energy to the powder mixture ${ }^{13}$. The objective of milling is to reduce the particle size and blending of particles in new phases. The different type of ball milling can be used for synthesis of nanomaterials in which balls impact upon the powder charge ${ }^{12}$.

High-energy ball milling is a convenient way to produce nanosized powders. It is the most common method reported in the literature for the synthesis of intermetallic nanoparticles. Before a mechanical milling is started, powder(s) is loaded together with several heavy balls (steel or tungsten carbide) in a container. By vigorously shaking or high-speed rotation, a high mechanical energy is applied on the powders because of collision with 
heavy balls ${ }^{10}$. A type of ball mill is shown in Figure $1^{12}$. Mechanical ball milling has been used to blend aluminum with magnesium and carbon in order to alter its chemical properties and combustion behavior ${ }^{14-16}$. The studies to make blends with magnesium used particles tens of micrometers in size $^{17}$.

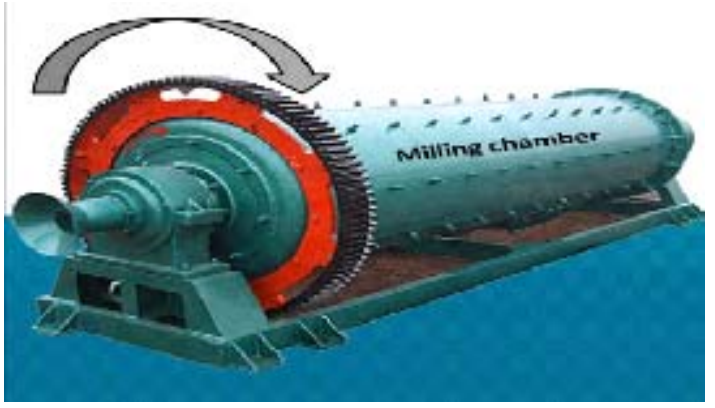

Fig. 1: A rock tumbler Ball mills (reprinted from Ref. 12)

Interestingly, aluminum nanoparticles ball milled with carbon were much more reactive than standard aluminum nanoparticles, and had to be handled under hexane to keep them from spontaneously combusting in air $^{18}$.

\section{Mechanochemical synthesis}

Mechanochemistry is the coupling of mechanical and chemical phenomena on a molecular scale and includes mechanical breakage and chemical behavior of mechanicallystressed solids. Mechanochemical synthesis differs from standard ball milling. A standard ball milling process under inert atmosphere results in a moderate reduction of powder particle size and eventually the formation of nanosized grains within micron-sized particles. The mechanochemical method involves the initiation of a solid-state displacement reaction during the ball milling process which can result in nanosized particles (down to $\sim 5 \mathrm{~nm}$ in size) embedded within larger by-product phase particles ${ }^{3}$.

In mechanochemical processes that utilize to change the chemical composition of precursors, the high energy ball milling equipments is generally used $^{13}$. The mechanochemical synthesis process has been used in the past to synthesize a broad range of metal nanoparticles (e.g. Ag, Co, Cr, Cu...) as well as other compounds such as oxides and sulphides. Particle size control can be gained by adjusting factors such as: the volume fraction of the by-product phase formed during milling, milling time, milling collision energy (ball-to-powder mass ratio and ball size), milling temperature, and the use of process control agents.

In 2009, a mechanochemical synthesis process has been used to synthesise aluminium nanoparticles. The aluminium is synthesised via a solid state chemical reaction which is initiated inside a ball mill at room temperature between either lithium $(\mathrm{Li})$ or sodium $(\mathrm{Na})$ metal which act as reducing agents with unreduced aluminium chloride (AICl3). The Al nanoparticles were 25$100 \mathrm{~nm}$ in dimensions as measured by TEM $^{3}$.

\section{Liquid-phase synthesis \\ Laser ablation}

Pulsed laser ablative deposition (PLD) is an attractive synthetic method owing to its ability to produce nanoparticles with a narrow size distribution and a low level of impurities ${ }^{19,20}$. Aluminum nanoparticles with diameters of tens to $500 \mathrm{~nm}$ of various shapes can be prepared by irradiating an aluminum foil with 50 fs pulses of a 0.8 ìm wavelength laser beam ${ }^{21}$.

Three main steps contribute in laser ablation synthesis method and formation of nanoparticles from a target immersed in liquid. Laser pulse, first, heats up the target surface to the boiling point, and thus, plasma plume containing vapor atoms of target is generated. Then, plasma expands adiabatically; and finally, nanoparticles will be generated when condensation occurs. Synthesis parameters such as laser wavelength, laser energy, pulse width, liquid media type, and ablation time can notably affect the product characteristics.

In 2010, Aluminum nanoparticles were synthesized by pulsed laser ablation of Al targets in ethanol, acetone, and ethylene glycol. Comparison between ethanol and acetone clarified that acetone medium leads to finer nanoparticles (mean diameter of $30 \mathrm{~nm}$ ) with narrower size distribution (from 10 to $100 \mathrm{~nm}$ ) ${ }^{5}$. Hur et al. report the synthesis of $\mathrm{Mg}-\mathrm{Al}$ and $\mathrm{Zn}$-Al-layered double 
hydroxides using the laser ablation in the liquid technique. Average diameters of these structures were about $500 \mathrm{~nm}$ and the thickness of a single layer was approximately about $6.0 \mathrm{~nm}^{22}$.

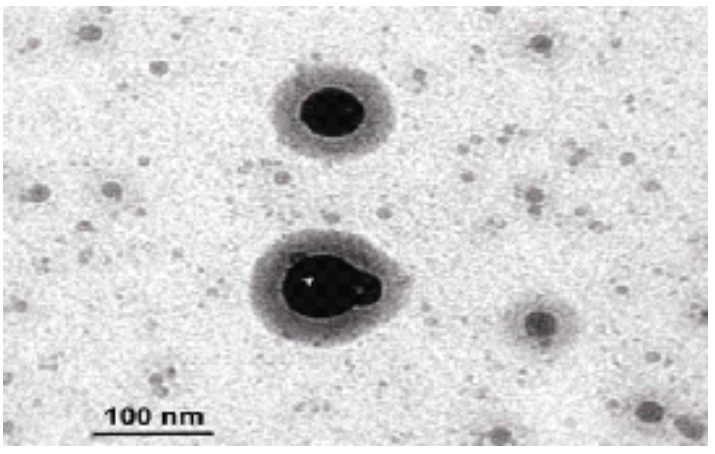

Fig. 2: TEM view of nanoparticles generated by ablation of bulk Al target in ethanol (Ref. 24)

In 2009, aluminium nanoparticles were produced by pulsed laser ablation of a sample of pure aluminium situated in distilled water. They provides the possibility to generate a large variety of nanoparticles that are free of both surfaceactive substances and counter-ions The sample was irradiated by the focused output of the third harmonics of pulsed nanosecond Nd : YAG laser operating at $10 \mathrm{~Hz}$ frequency. The typical thickness of the liquid above the target was $10 \mathrm{~mm}^{23}$. Stratakis et al. reported on Generation of Al nanoparticles via ablation of bulk $\mathrm{Al}$ in liquids with short laser pulses. The colloidal nanoparticles solutions obtained with fs pulses exhibit a yellow coloration and show an increased optical absorption between 300 and 400 $\mathrm{nm}$, tentatively assigned to the plasmon resonance of nanosized Al. Generated Al nanoparticles exhibit minimal oxide cladding and were pretty stable as they became slowly oxidized by air oxygen. The average size of Al nanoparticles formed lies between 10 and $60 \mathrm{~nm}$, depending on the experimental conditions. This is illustrated in Fig. 2 where the NPs generated using 150 ps pulses have a core-shell structure with metallic core. On the other hand presence of an oxide layer is well seen on TEM images of NPs produced by ablation with ps pulses without using anaerobic conditions [24]. In 2012, it was investigated the viability of laser ablation of $\mathrm{W}$ and $\mathrm{Al}$ metal targets immersed in acetone and water, respectively, as a technique to produce metal nanoparticles. The setup used for the synthesis of metal particles by laser ablation of a metal target in liquid medium is presented in Figure 3. Particles size distributions peak were relatively narrow, peaking at $50-70 \mathrm{~nm}^{25}$.

One problem with this method is that in long ablation times, the ablation rate decreases. It occurs when high concentrations of nanoparticles in produced colloidal solution blocks the laser path, and thus, a part of laser energy is absorbed by formerly synthesized nanoparticles instead of the target surface ${ }^{5}$

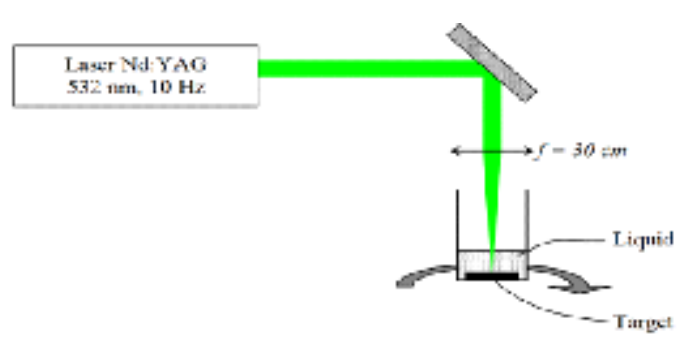

Fig. 3: Schematic representation of the experimental setup (laser ablation) (Ref. 25)

\section{Exploding wire}

Electro-explosion of metal wires has only recently been seriously applied to make aluminum nanoparticles. In electro-explosion, a brief but powerful current pulse creates an electromagnetic field around the wire that holds it together while it is superheated to tens of thousands of degrees. When the current ceases, the electromagnetic field disappears and the wire fragments into nanosized particles. The shapes and sizes of the resulting particles depend on many factors, such as the shape and size of the wire, the voltage, and the nature of the electrical pulse.

Sen et al. described a process for the production of nanoparticles of $\mathrm{Cu}, \mathrm{Ag}, \mathrm{Fe}$ and $\mathrm{Al}$ which involves exploding their respective wires, triggered by large current densities in the wires. The explosion was carried out in a dense medium, typically water or some heavy alcohol where the particles remain suspended and is collected in the following manner. An initial centrifuge of the suspension at 5000 RPM separates the fluid from the solid mass. While the former is rejected, the solid mass is dispersed in electronic grade acetone ${ }^{26}$. 


\section{Solution reduction}

In this method, it is used from a reducing agent for the reduction of $\mathrm{Al}^{+3}$ ions in solution. The chemical reduction route is simple and not time consuming. It has also an immense potential to scale up when required to meet mass manufacturing needs. Treatment of aluminum chloride with lithium aluminum hydride in mesitylene at $164^{\circ} \mathrm{C}$ affords aluminum nanoparticles. The nanoparticle aggregates made by this method were $110-210$ $\mathrm{nm}$ in diameter. This method proved to be inconvenient for scale up and, even after being washed, the nanoparticles still contained measurable levels of carbon, oxygen, and chlorine ${ }^{27}$.

In 2012, Al nanoparticles were synthesized by solution reduction process successfully. They used from benzildiethylenetriamine as a reducing agent in methanol, ethanol, water, acetonitrile, cyclohexane and dimethylsulphoxide. The best results obtained by the ethanol for the synthesis of Al nanoparticles in the range of $4-13 \mathrm{~nm}$. The influences of parameters on the size of $\mathrm{Al}$ nanoparticles were studied and the referential process parameters were obtained ${ }^{2}$. Also Al nanoparticles (5-8 nm size) have been synthesized by using $\mathrm{NaBH} 4$ or $\mathrm{LiAlH} 4{ }^{45}$. In another study, it reported the synthesis of $\mathrm{Al} / \mathrm{Au}$ bimetallic nanoparticles in water solution. They used from $\mathrm{Al}^{+3}$ and $\mathrm{Au}^{+3}$ metal salts and reducing solution contain sodium citrate, tannic acid, and sodium carbonate ${ }^{28}$.

In 2012, the synthesis of aluminium nanoparticles in a polypropylene (PP) matrix by a sol-gel process in the melt was investigated. Their work confirms that it is possible to produce inorganic nanoparticles in a polymeric matrix by reaction in the molten state without solvents ${ }^{29}$.

Generally in chemical reduction method, reducing agent is a chemical solution such as benzildiethylenetriamine, lithium aluminum hydride and etc.

\section{Decomposition process}

The chemical route based on thermal and/ or catalytic decomposition of alane in the presence of a surface passivation agent for particle protection and stabilization has been identified as being particularly promising. The passivation agent for $\mathrm{Al}$ nanoparticles could be a metal coating or organic molecules such as perfluorinated carboxylic acids, which could also serve as an oxidant source under energetic conditions. This method has generally yielded Al particles of $50-200 \mathrm{~nm}$ in average sizes, though smaller particles have been obtained recently in sonochemical environment with oleic acid as the surface passivation agent ${ }^{6}$. Li et al. reported the use of nanoscale cavities in perfluorinated ionomer membrane as templates for the facile synthesis of small Al nanoparticles (diameters on the order of $10 \mathrm{~nm}$ ) via catalytic decomposition of an alane precursor ${ }^{6}$. Aluminum nanoparticles can be obtained on a larger scale by decomposing isolated samples of the alane amine adduct $\mathrm{H} 3 \mathrm{Al}(\mathrm{NMe} 2 \mathrm{Et})$ in mesitylene at $164{ }^{\circ} \mathrm{C}$. The aluminum nanoparticles aggregates synthesized by this method were with diameters of $44-82 \mathrm{~nm}^{27}$. In another study, pure $\mathrm{Mg}-\mathrm{Al}$ alloys and $\mathrm{Ni}$ nanoparticles prepared by thermal decomposition on bipyridyl complex of metals ${ }^{30}$.

In 2009, the synthesis of aluminum nanoparticles was investigated systematically using dimethylethylamine alane and 1-methylpyrrolidine alane as precursors and molecules with one or a pair of carboxylic acid groups as surface passivation agents. Meziani et al. found that the passivation agent played dual roles of trapping aluminum particles to remain them nanoscale during the alane decomposition and protecting the aluminum nanoparticles from surface oxidation ${ }^{31}$.

\section{Gas-phase synthesis \\ Gas evaporation}

The most common method to synthesize aluminum nanoparticles is the evaporation of aluminum from the molten state into a chamber filled with an inert gas, where the gaseous metal condenses. The purity of the aluminum starting material, and the type and purity of the inert gas atmosphere, strongly influence the properties of the aluminum nanoparticles obtained ${ }^{32,33}$. A modified inert gas evaporation method called cryomelting can also be used to make aluminum nanoparticles ${ }^{34}$. In the cryomelting process, the evaporated metal is rapidly condensed in region cooled to about $70 \mathrm{~K}$. This method can produce 20 
- $500 \mathrm{~nm}$ aluminum nanoparticles in which $60 \%$ of the particles are smaller $70 \mathrm{~nm}$ in size, as observed by $\mathrm{TEM}^{34}$. In another research, the thermal behavior of aluminum nanoparticles prepared by inert gas condensation process was investigated ${ }^{35}$.Also Al nanoparticles were prepared by the inert gas condensation method by Fernández et al. It was found the presence of an alumina overlayer of approximately $4 \mathrm{~nm}$ covering the aluminium nanoparticles (23 $\mathrm{nm}$ in diameter) ${ }^{36}$.

In 2010, a novel electromagnetic levitational gas condensation (ELGC) system was designed and manufactured for the synthesis of aluminum nanoparticles. It was found that the best argon flow rate for the synthesis of aluminum nanoparticles was found about 10-15 lit/min ${ }^{37}$.

\section{Exploding wire}

Wire explosion is basically a top-down approach to produce metallic nano-powders. A pulsed discharge system is used to supply a high power pulsed current to a thin metal wire and lead to the wire explosion. Large amount of heat from Joule heating will be dissipated in the wire to melt, evaporate and subsequently ionize it. Plasma formed during the process expands due to its high temperature and high density. This plasma will be rapidly cooled during expansion when it interacts with the surrounding gas and nanoparticles will be formed through nucleation process. Yap et al. (2008) reported exploding wire discharge for synthesis of Al nanoparticles. The Al nanoparticles were less than $100 \mathrm{~nm}$ in dimensions as measured by SEM images. The schematic of the wire explosion chamber is shown in Fig. ${ }^{38}$. In another study, nano aluminium particles were produced by wire explosion process (WEP) in nitrogen, argon and helium atmospheres. The relationship between size of the particle generated in the explosion process and the type of inert gas/pressure was analysed. It is realized that energy deposited to the conductor and duration of current flow have major impact on particles produced by this process ${ }^{39}$. In 2012, an experimental device based on the electrical explosion of metallic wires for the nanopowders production and collection was designed and built. Also, aluminum nanopowders were produced by electrical exploding aluminum wire and collected by the microporous membrane filter successfully under different experimental conditions ${ }^{40}$. Also Sindhu et al. proposed a modelling of the nanoparticles formation in the wire explosion process. It was found that the plasma formed during the explosion plays a major role in the particle formation, and the modelling studies confirm that particle formation is not an instantaneous process but requires a certain time period to form stable sizes and shapes ${ }^{41}$.

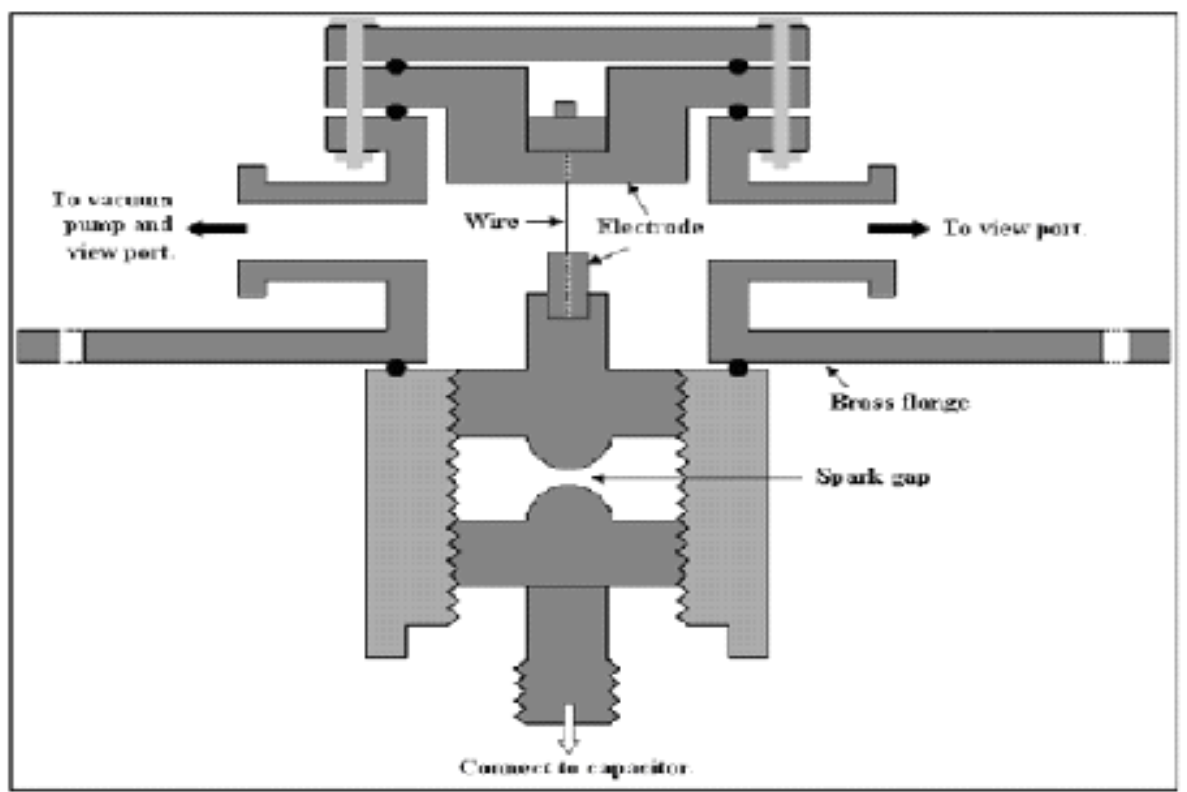

Fig. 4: Schematic of explosion chamber and the exploding wire (Ref. 38) 
Generally the wire explosion technique has several advantages for the generation of nanoparticles. First, the energy deposition can be controlled precisely, and second, the discharge energy is deposited into the wire with high efficiency and the wire is exploded extremely quickly to form a supersaturated vapor ${ }^{38}$.

\section{Laser ablation}

As mentioned above, pulsed laser ablation (PLA) is an attractive synthetic method owing to its ability to produce nanoparticles with a narrow size distribution. In the view of gas dynamics, the PLA process can be classified into (i) evaporation of the target material and (ii) hydrodynamic expansion of the ablated plume into the ambient gas. In nanoparticle formation, the following stages must be considered: (i) homogeneous nucleation, where vapor atoms produced by laser ablation have been supersaturated, and (ii) particle growth, where the critical nuclei are growing, capturing atoms on their surfaces, and making the transition into large particles $^{42}$.

Aluminum nanoparticles were synthesized using laser ablation method in argon gas as ambient gas by Yamamoto et al. They found that it is possible to control the particle size synthesized by controlling the ambient gas temperature ${ }^{4}$. In addition, Al nanoparticles generated by laser ablation can be coated with carbon by introducing ethylene to the argon quench flow. The resulting nanoparticles have an average mobility diameter of $80 \mathrm{~nm}^{43}$. In 2011, the process of nanoparticle generation during nanosecond and picosecond laser ablation of various metals ( $\mathrm{Ni}, \mathrm{Al}$, $\mathrm{W}$ and stainless steel) in ambient air and argon gas was investigated. It was found that the size distribution and number concentration of generated metal particles during the laser ablation in ambient air differed from those in argon gas medium. The number concentrations of generated nanoparticles during the laser ablation in argon gas, compared to the produced nanoparticle concentrations in ambient air, were up to 100 times higher ${ }^{44-46}$.

\section{CONCLUSIONS}

Al nanoparticles are one of the most important nanoparticles because of their applications. These nanoparticles have many important applications that include: pyrotechnic, propellant, explosive industries, rocket fuel, igniter, smokes, tracers, alloy powder metallurgy parts for automobiles and aircrafts, heat shielding coatings of aircrafts, corrosion, resistant, conductive and heat reflecting paints, conductive and decorative plastics, soldering and termite welding. Application of $\mathrm{Al}$ nanoparticles in these fields is dependent on the ability to synthesize particles with different chemical composition, shape, size, and monodispersity. Generally, there are various methods to synthesize Al nanoparticles. The techniques for synthesizing aluminum nanoparticles can be divided into solidphase, liquid-phase and gas-phase processes. Nevertheless high purity powders and nanopowders of active metals such as Al are not easily synthesized in as much as their rapid oxidation occurs easily. The small sizes of aluminum nanoparticles make them particularly susceptible to excessive oxidation while being stored prior to use. As a result, much attention has been devoted to modifying the aluminum nanoparticles in order passivate the surface against the formation of an oxide overlayer, and thereby obtain longer shelf lives and better burn properties.

\section{REFERENCES}

1. Mandal, D., Bolander, M.E., Mukhopadhyay, D., Sarkar, G., Mukherjee, P., Appl Microbiol Biotechnol. 2006, 69, 485.

2. 5- Sulekh Chandra, Avdhesh Kumar, Praveen Kumar Tomar., Thermal and spectral studies. Spectrochimica Acta Part A, 2012, 92, 392- 397.
3. M. Paskevicius, J. Webbb, M.P. Pitt, T.P. Blach, B.C. Hauback, E.MacA. Gray, C.E. Buckley, Journal of Alloys and Compounds 2009, 481, 595-599.

4. R. A. Ganeev, G. S. Boltaev, R. I. Tugushev, T. Usmanov, Applied Physics A 2010, 100, 119-123. 
5. Arash Baladi, Rasoul Sarraf Mamoory, Applied Surface Science 2010, 256, 75597564.

6. Heting Li, Mohammed J. Meziani, Fushen Lu, Christopher E. Bunker, Elena A. Guliants, and Ya-Ping Sun, J. Phys. Chem. C 2009, 113, 20539-20542.

7. Gardea-Torresdey, J.L., Gomez, E., PeraltaVidea, J.R., Parsons, G.J., Troiani, H., JoseYacama, M., Langmuir 2003, 19, 1357.

8. Charinpanitkul, T., Faungnawakij, K., Tanthapanichakoon, W., Adv Powder Technol 2008, 19, 443.

9. Sharma, V.K., Yngard, R.A., Lin, Y., Adv Colloid Interfac 2009, 145, 83.

10. Tavakoli, A., Sohrabi, M., Kargari, A., Chem. Pap. 2007, 61, 151.

11. Mohanpuria, P., Rana, N.K., Yadav, S.K., J Nanopart Res 2008, 10, 507.

12. Thakur Prasad Yadav, Ram Manohar Yadav, Dinesh Pratap Singh, Nanoscience and Nanotechnology 2012, 2, 22-48.

13. Boldyrev, V.V. \& Tkacova, K., Journal of Materials Synthesis and Processing 2000, 8, 121-132.

14. Shoshin, Y. L.; Mudryy, R. S.; Dreizin, E. L. Combust. Flame 2002, 128, 259-269.

15. Dreizin, E. L.; Shoshin, Y.L.; Mudryy, R. S. CPIA Publication 2001, 705, 189-199.

16. Pivkina A., Streletskii A., Kolbanev I., Ul'yanova P., Frolov Y., Butyagin P., Schoonman J., J. Mater. Sci. 2004, 39, 54515453.

17. Streletskii A. N., Pivkina A. N., Kolbanev I. V., Borunova A. B., Leipunskii I. O., Pshechenkov P. A., Lomaeva S. F., Polunina I. A., Frolov Y. V., Butyagin P.Y., Colloid J. 2004, 66, 736-744.

18. Streletskii A. N., Kolbanev I. V., Borunova A. B., Butyagin P. Y., J. Mater. Sci. 2004, 39, 5175-5179.

19. Pronko P. P., Zhang Z., VanRompay P. A., App. Surf. Sci. 2003, 208, 492-501.

20. Ullmann M., Friedlander S. K., Schmidt-Ott A. J., Nanopart. Res. 2002, 4, 499-509.

21. Eliezer S., Eliaz N., Grossman E., Fisher D., Gouzman I., Henis Z., Pecker S., Horovitz Y., Fraenkel M., Maman S., Lereah Y., Phy. Rev. 2004, 69, 144119/1-144119/6.

22. Tae-Bong Hub, Tran X. Phuoc, Minking K. Chyu, Optics and Lasers in Engineering
2009, 47, 695-700.

23. V. Damian, C. Udrea, M. Bojan, C. Luculescu, A. Armaselu, I. Apostol, Proceedings of SPEI 2011, 8001, 1.

24. Emmanuel Stratakis, Marios Barberoglou, Costas Fotakis, Guillaume Viau, Cecile Garcia, and Georgy A. Shafeev, Optics Express 2009, 17, 12650-12659.

25. F. Stokker-Cheregi, T. Acsente, I. Enculescu, C. Grisolia, G. Dinescu, Digest Journal of Nanomaterials and Biostructures. 2012, 7, 1569-1576.

26. P Sen, Joyee Ghosh, Alqudami Abdullah, Prashant Kumar, Vanada, Proc. Indian Acad. Sci. (Chem. Sci.) 2003, 115, 499-508.

27. Haber J. A., Buhro W. E., J. Am. Chem. Soc. 1998, 120, 10847-10855.

28. M. Gilaki, Pakistan Journal of Biological Sciences 2010, 13, 809-813.

29. M. Oliveira, R. Nogueira, A.V. Machado, Reactive \& Functional Polymers 2012, 72 , 703-712.

30. N. A. Niaz, Ishaq Ahmad, S. Nasir, Z. Wazir, R. Hussain, N. R. Khalid, S. Tajammul Hussain, Digest Journal of Nanomaterials and Biostructures 2013, 8, 423 - 431.

31. Meziani MJ, Bunker CE, Lu F, Li H, Wang W, Guliants EA, Quinn RA, Sun YP, ACS Appl Mater Interfaces. 2009, 1, 703-709.

32. Sun X. K., Xu J., Chen W. X., Wei W. D., Nanostruct. Mater. 1994, 4, 337-44.

33. Puszynski J. A., Proc. Int. Pyrotech. 29th, 2002, 191-202.

34. Champion Y., Bigot, J. Nanostruct. Mater. 1998, 10, 1097-1110.

35. Sanchez-Lopez J. C., Fernandez A., Conde C. F., Conde A., Morant C., Sanz, J. M., Nano. Mat. 1996, 7, 813-822.

36. Fernández A, Sánchez-López JC, Caballero A, Martin JM, Vacher B, Ponsonnet L., J Microsc. 1998, 191, 212-220.

37. Kermanpur A., Dadfar MR, Nekooei Rizi B, Eshraghi M., J Nanosci Nanotechnol. 10, 2010, 6251-6255.

38. L Yap, Y S Lee, W H Tay, C S Wong, Proceedings of the International Workshop On Plasma Computations \& Applications (IWPCA2008) 60-64.

39. T. K. Sindhu, R. Sarathi, S. R. Chakravarthy, Bulletin of Materials Science 2007, 30, 187- 
195.

40. Liu Longchen, Zhang Qiaogen, Zhao Junping, Wang Zhe, Zhang Yu, Liu Xuandong, Plasma Science (ICOPS) 2012, 2P-101

41. TK Sindhu, R Sarathi and S R Chakravarthy, Nanotechnology 2008, doi:10.1088/09574484/19/02/025703

42. Han M., Gong Y., Zhou J., Yin C., Song F., Muto M., Takiya T., Iwata Y., Phys. Lett., 2002, A 302, 182-189.
43. Park K., Rai A., Zachariah M. R., J. Nanopart. Res. 2006, 8, 455-464.

44. V. Dudoitis, V. Ulevièius, G. Raèiukaitis, N. Đpirkauskaitë, K. Plauðkaitë, Lithuanian Journal of Physics 2011, 51, 248-259.

45. Vemal Raja Manikam, Kuan Yew Cheong, Khairunisak Abdul Razak, Materials Science and Engineering:B 2011, 176, 187-203.

46. Guoqiang, Z. and Wenying, W., Orient J. Chem., 2012, 28(2), 651-655. 